\title{
Fatness, Fitness, and Cardiometabolic Risk Factors among Sixth-Grade Youth
}

\begin{abstract}
RUSSELL JAGO ${ }^{1}$, KIMBERLY L. DREWS ${ }^{2}$, ROBERT G. MCMURRAY 3 , DEBBE THOMPSON $^{4}$, STELLA L. VOLPE ${ }^{5}$, ESTHER L. MOE ${ }^{6}$, JOHN M. JAKICIC ${ }^{7}$, TRANG H.

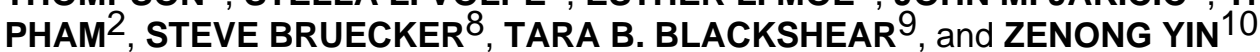

${ }^{1}$ Department of Exercise, Nutrition \& Health Sciences, University of Bristol, Bristol, England, UNITED KINGDOM
\end{abstract}

${ }^{2}$ The George Washington University Biostatistics Center, Washington, DC

${ }^{3}$ Department of Exercise \& Sport Science, University of North Carolina at Chapel Hill, Chapel Hill, $\mathrm{NC}$

${ }^{4}$ USDA/ARS Children's Nutrition Research Center, Baylor College of Medicine, Houston, TX

${ }^{5}$ Division of Biobehavioral and Health Sciences, University of Pennsylvania, School of Nursing, Philadelphia, PA

Copyright (C) 2010 by the American College of Sports Medicine

Address for correspondence: Russell Jago, Ph.D., Department of Exercise, Nutrition and Health Sciences, Centre for Sport, Exercise and Health, University of Bristol, Tyndall Ave., Bristol, England, BS8 1TP, United Kingdom; russ.jago@gmail.com..

The writing group for this article was the following: R. Jago (chair), K. L. Drews, R. G. McMurray, D. Thompson, S. L. Volpe, E. L. Moe, J. M. Jakicic, T. H. Pham, S. Bruecker, T. B. Blackshear, and Z. Yin.

The HEALTHY Study Group (* indicates principal investigator or director):

STOPP-T2D study chair

Children's Hospital Los Angeles: F. R. Kaufman

Field centers

Baylor College of Medicine: T. Baranowski*, L. Adams, J. Baranowski, A. Canada, K. W. Cullen, M. H. Dobbins, R. Jago, A.

Oceguera, A. X. Rodriguez, C. Speich, L. T. Tatum, D. Thompson, M. A. White, and C. G. Williams

Oregon Health \& Science University: L. Goldberg*, D. Cusimano, L. DeBar, D. Elliot, H. M. Grund, K. Kuehl, S. McCormick, E.

Moe, J. B. Roullet, A. Sleigh, and D. Stadler

Temple University: G. D. Foster* (Steering Committee Chair), J. Brown, B. Creighton, M. Faith, E. G. Ford, H. Glick, S. Kumanyika,

J. Nachmani, J. Rosen, L. Rosen, S. Sherman, S. Solomon, A. Virus, S. L. Volpe, and S. Willi

University of California at Irvine: D. Cooper*, S. Bassin, S. Bruecker, D. Ford, P. Galassetti, S. Greenfield, J. Hartstein, M. Krause,

N. Opgrand, Y. Rodriguez, and M. Schneider

University of North Carolina at Chapel Hill: J. S. Harrell*, A. Anderson, T. Blackshear, J. Buse, J. Bridgman, A. Gerstel, C. Giles, W. Hall, A. Jessup, P. Kennel, R. Matthews, R. G. McMurray, D. Rubin, A. M. Siega-Riz, M. Smith, A. Steckler, A. Stringer, and A.

Zeveloff

University of Pittsburgh: M. D. Marcus*, M. Carter, S. Clayton, B. Gillis, K. Hindes, J. M. Jakicic, R. Meehan, R. Noll, T. Songer, J.

Vanucci, and E. M. Venditti

University of Texas Health Science Center at San Antonio: R. Treviño*, A. Garcia, D. Hale, A. E. Hernandez, I. Hernandez, C.

Mobley, T. Murray, K. Surapiboonchai, and Z. Yin

Other investigators

K. Resnicow, University of Michigan; M. Goran, University of Southern California; M. Engelgau, L. Y. Wang, and P. Zhang, Centers for Disease Control and Prevention

Coordinating center

George Washington University: K. Hirst*, K. L. Drews, S. Edelstein, L. El ghormli, L. S. Firrell, M. Huang, P. K. Feit, S. L. Mazzuto,

T. Pham, and A. Wheeler

Project office

National Institute of Diabetes and Digestive and Kidney Diseases: B. Linder*, C. Hunter, M. Staten

Central blood laboratory

University of Washington Northwest Lipid Metabolism and Diabetes Research Laboratories: S. M. Marcovina*

No conflicts are reported.

The results of the present study do not constitute endorsement by the American College of Sports Medicine. 
${ }^{6}$ Division of Health Promotion and Sports Medicine, Oregon Health \& Sciences University, Portland, OR

${ }^{7}$ Department of Health and Physical Activity, University of Pittsburgh, Pittsburgh, PA

${ }^{8}$ Department of Health and Physical Activity, University of California at Irvine, Irvine, CA

${ }^{9}$ Health and Physical Education, Cairo American College, Cairo, EGYPT

${ }^{10}$ Department of Health and Kinesiology, University of Texas at San Antonio, San Antonio, TX

\section{Abstract}

Purpose-Examine whether cardiometabolic risk factors are predicted by fitness or fatness among adolescents.

Methods-Participants are 4955 (2614 female) sixth-grade students with complete data from 42 US middle schools. Fasting blood samples were analyzed for total cholesterol, HDL- and LDLcholesterol, triglyceride, glucose, and insulin concentrations. Waist circumference and blood pressure were assessed. Body mass index (BMI) was categorized as normal weight, overweight, or obese as a measure of fatness. Fitness was assessed using the multistage shuttle test and was converted into gender-specific quintiles. Gender-specific regression models, adjusted for race, pubertal status, and household education, were run to identify whether BMI group predicted risk factors. Models were repeated with fitness group and both fitness and fatness groups as predictors.

Results-Means for each risk factor (except HDL, which was the reverse) were significantly higher $(P<0.0001)$ with increased fatness and differed across all BMI groups $(P<0.001)$. Waist circumference, LDL-cholesterol, triglycerides, diastolic blood pressure, and insulin were inversely associated with fitness $(P<0.001)$. When both fatness and fitness were included in the model, BMI was associated $(P<0.001)$ with almost all cardiometabolic risk factors; fitness was only associated with waist circumference (both genders), LDL-cholesterol (males), and insulin (both genders). Other associations between fitness and cardiometabolic risk factors were attenuated after adjustment for BMI group.

Conclusions-Both fatness and fitness are associated with cardiometabolic risk factors among sixth-grade youth, but stronger associations were observed for fatness. Although maintaining high levels of fitness and preventing obesity may positively affect cardiometabolic risk factors, greater benefit may be obtained from obesity prevention.

\section{Keywords}

FIT; OBESITY; CHILDREN; ADOLESCENTS; CARDIOVASCULAR DISEASE

Data from the 1999-2002 US National Health and Nutrition Examination Survey indicated that less than one-third of 12- to 19-yr-old adolescents in the United States met fitness recommendations (28). Low aerobic fitness levels have been associated with increased risk for cardiovascular diseases (21) and diabetes mellitus among adults (23). In children and adolescents, low aerobic fitness has also been associated with cardiometabolic (5) risk factors (large waist circumference, high blood pressure, and high insulin, glucose, and lipid levels) (15). Data from 9- and 15-yr-old youths participating in the European Youth Heart Study indicated that low aerobic fitness was associated with increased waist circumference, insulin, glucose, and lipid concentrations and a clustered score that included all of the cardiometabolic risk factors (13). Interestingly, the researchers also found that these associations were independent of both physical activity (the behavior that influences fitness) and fatness (13). 
Extensive research has shown that the risk of cardiovascular disease and diabetes among overweight adults is ameliorated if the adult has a high level of fitness $(22,32)$. For example, data from the Cooper Clinic in Dallas showed that unfit, lean men had double the risk of cardiovascular disease and all-cause mortality than lean men who were fit (22). Moreover, unfit lean men had a higher risk of cardiovascular disease and all-cause mortality than fit but overweight men (22). These findings suggest that fitness offers a degree of protection against cardiovascular disease and mortality even if the individual is overweight. Similar findings have been reported for women (32). Although there is a lack of evidence about the associations among childhood fitness, adiposity, and mortality, the prevalence of cardiometabolic risk factors has been shown to increase with higher levels of obesity among youth $(20,31)$. Higher levels of obesity among children and adolescents have also been associated with low levels of aerobic fitness (16). Comparable associations among fitness, adiposity, and cardiometabolic risk factors findings have also been reported among a relatively small (296 boys and 188 girls) sample of 8- to 18-yr-old US youths (12). It is not clear if associations are evident for all cardiometabolic risk factors among adolescents. Understanding the importance of fitness, obesity status, and the prevalence of cardiometabolic risk factors among adolescents will provide key information to aid the design of strategies to reduce the prevalence of cardiometabolic risk factors among adolescents, thereby reducing disease risk.

Boys have higher levels of aerobic fitness than girls (28), with some evidence of ethnic differences in fitness levels (16). Maturation differences in both fitness (28) and cardiometabolic risk factors have also been reported (39). Thus, in examining the associations among fitness, obesity, and cardiometabolic risk factors, it is important to account for these biological and sociodemographic factors. It is also important to recognize that the majority of previous studies that have examined associations between aerobic fitness and cardiometabolic risk factors among youth have reported fitness in relation to body mass $(\mathrm{mL}$ $\mathrm{O}_{2} \cdot \mathrm{kg}^{-1} \cdot \mathrm{min}^{-1}$ or $\left.\mathrm{W} \cdot \mathrm{kg}^{-1}\right)(4,30)$, which precludes the necessary examination of the independent effects of fitness and fatness. In light of the evidence presented above, the aim of this article was to examine the extent to which fitness and obesity status are independently associated with cardiometabolic risk factors among a large, ethnically diverse sample of sixth-grade US adolescents.

\section{METHODS}

\section{Sample}

Data were collected as part of the baseline assessment of the HEALTHY Study, a National Institute of Diabetes and Digestive and Kidney Diseases multisite study designed to reduce the prevalence of risk factors for type 2 diabetes mellitus among middle school children $(7,18)$. Data were collected at seven field centers (Baylor College of Medicine, Houston, TX; Oregon Health \& Science University, Portland, OR; University of California at Irvine, Irvine, CA; Temple University, Philadelphia, PA; University of North Carolina at Chapel Hill, Chapel Hill, NC; University of Pittsburgh, Pittsburgh, PA; and University of Texas Health Science Center at San Antonio, San Antonio, TX), with a coordinating center in Washington, DC (George Washington University Biostatistics Center). Participants were recruited from 42 middle schools, 6 per field center. Schools were required to have at least $50 \%$ of students eligible for free or reduced-price lunch or belonging to a minority group and an annual student attrition rate from all causes $\leq 25 \%$. All sixth-grade students were invited to participate in a "health screening." Students were given a $\$ 50$ incentive for data collection. This study was approved by the institutional review boards at each field center, and written informed parental consent and child assent were obtained. 


\section{Procedures}

Measurements were conducted by personnel who were trained and certified in recording height, weight, and waist circumference accurately. Height and body mass were measured without shoes using the Prospective Enterprises PE-AIM-101 stadiometer and the SECA Corporation Alpha 882 electronic scale. Body mass index (BMI; $\mathrm{kg} \cdot \mathrm{m}^{-2}$ ), which provides an index of overweight or obesity, was calculated and, for the purposes of this article, was interpreted as an indicator of "fatness." Waist circumference was taken using a Gulick tape measure (G-tape) with a tension device on bare skin measured just above the iliac crest. Blood pressure was recorded three times using an automated blood pressure monitor (HEM-907XL; Omron, Vernon Hills, IL). The initial value was recorded after the participant had been seated quietly for 5 min with each subsequent value recorded $1 \mathrm{~min}$ after the preceding recording. An appropriate size cuff was used for all participants. The mean of the second and third recordings was used in all subsequent analyses.

Pubertal status was self-reported individually in private using the Pubertal Development Scale (29) and was converted to pubertal stage groups that are consistent with the five pubertal stages that have been outlined by Tanner (33). Ethnicity and race were collected by student self-report in response to two separate items. Frequently, this age group did not understand the distinction between ethnicity and race, and the information from both items was combined and categorized as follows: anyone checking "Hispanic or Latino" ethnicity was classified as Hispanic, non-Hispanics choosing only "black or African American" race were classified as black, non-Hispanics choosing only "white" race were white, and all other response categories were combined into "other." A parent or guardian completed a questionnaire providing the highest level of education in the household. On the basis of these responses, the highest household education level was classified as low (less than high school or some high school), middle (high school graduate or some college or specialized training), or high (college or university graduate or postgraduate training or degree).

Fitness was assessed using the 20-m shuttle test (20-MST) (24,25), most frequently during a physical education class. The test required students to run back and forth between two lines set $20 \mathrm{~m}$ apart. The running pace was determined by audio signals emitted from a prerecorded CD. The test started at $8.5 \mathrm{~km} \cdot \mathrm{h}^{-1}$ and increased by $0.5 \mathrm{~km} \cdot \mathrm{h}^{-1}$ with each subsequent level. The test was completed when the participant was not able to complete the distance at the stipulated pace on two laps. A score for the test was based on the number of laps completed, and for the reasons outlined above, this score was not adjusted for body mass.

Participants were called the night before data collection to remind them not to eat or drink anything but water after midnight and were asked at the beginning of the blood screening when they last ate. Participants who reported eating after midnight were considered nonfasting and asked to return another day. Experienced pediatric phlebotomists obtained fasting blood samples that were processed and then shipped to the central blood laboratory at the University of Washington Northwest Lipid Research Laboratories for all analyses. Analyses of glucose were performed on a Roche P module autoanalyzer by the hexokinase method using reagent from Roche Diagnostics (Indianapolis, IN). Insulin was measured by a two-site immunoenzymometric assay performed using a Tosoh 1800 autoanalyzer (Tosoh Biosciences Inc., San Francisco, CA). The assay sensitivity level was $2.0 \mu \mathrm{U} \cdot \mathrm{mL}^{-1}$, with interassay and intra-assay coefficients of variation $<10 \%$. The assay had a high specificity as cross-reactivity with human C-peptide and pro-insulin was $0 \%$ and $2 \%$, respectively. Measurements of total plasma cholesterol, cholesterol in the lipoprotein fractions, and triglycerides were performed enzymatically on the Roche Modular-P autoanalyzer using methods standardized to the Centers for Disease Control and Prevention reference methods (37). Determination of HDL-cholesterol was performed after precipitation of apolipoprotein 
B-containing particles by dextran sulfate $\mathrm{Mg}^{2+}$. LDL-cholesterol was calculated using the equation of Fridewald et al. (14). This approach for calculating LDL is clinically reliable if the measurements of total and HDL-cholesterol are performed with a high level of accuracy and triglycerides are $<400 \mathrm{mg} \cdot \mathrm{dL}^{-1}$ (38). In the case of elevated triglycerides, a complete lipoprotein separation by ultracentrifugation, which allows quantization of the individual lipoprotein classes, was performed using the Lipid Research Clinics Beta Quantification procedure (17). The interassay coefficients of variations are consistently $<1.5 \%$ for total cholesterol and triglycerides and $<2 \%$ for HDL-cholesterol.

\section{Statistical analysis}

A total of 6358 sixth-grade students participated in the HEALTHY Study at baseline. Of these participants, 5394 had complete data for anthropometric assessments, blood values, 20-MST, pubertal stage survey, and parental or guardian report of highest level of household education. However, 439 of these participants reported other or mixed race and were dropped from all analysis because the results for this ethnic group are too heterogeneous to interpret. Therefore, analyses are presented for the remaining 4955 participants (2614 females). Pubertal data were collapsed into three groups, prepubertal (stage 1), early pubertal (stages 2 and 3), and pubertal (stages 4 and 5).

Descriptive statistics with means and SE for continuous measures and frequency distribution for categorical variables are presented in Table 1, both overall and by gender for the complete sample listed above. Fitness category was determined by quintiles on the basis of the number of laps for both genders, which are inexact because of ties. BMI percentile by age and gender was calculated using the Centers for Disease Control and Prevention 2000 criteria $(8,9)$. Youth with BMI $\geq 85$ th but $<95$ th percentile were classified as overweight, and those with BMI $\geq 95$ th percentile were classified as obese (1). In this study, the three-level BMI-based obesity status is used as a proxy of fatness.

Separate regression models were fitted for each continuous cardiometabolic risk factor using the PROC GLIMMIX procedure with either fitness or BMI group as an exposure measure. To adjust for the clustering of participants within schools, a random effect for school was included in the models. Because one-way ANOVA tests indicated that there were gender differences for both fitness $(P<0.0001)$ and BMI categories $(P<0.0013)$, models were run separately for males and females. All models were adjusted for pubertal stage group, race, and household education. Adjusted least-square means and 95\% confidence intervals were obtained from the model, with the overall $P$ values for a test of group differences (Tables 2 and 3). Where there were statistically significant group differences $(P<0.01)$, pairwise comparisons were carried out to determine which differences between categories were statistically significant. To account for multiple testing, Bonferroni $P$ value adjustments were performed for all pairwise comparisons.

Models including both BMI and fitness categories were fitted to examine whether their concurrent relationships with each cardiometabolic risk factor changed from the initial sets of models where BMI and fitness categories were entered into the model separately (Table 4). The models were adjusted for pubertal stage group, race, and household education. To aid in the interpretation of each model, overall $F$ test statistic values are presented (Table 4). As another measure of model fit, the Akaike Information Criterion (AIC) is also presented (Table 4). The AIC is AIC $=2 k-2 \ln (L)$, where $k$ is the number of parameters in the statistical model and $L$ is the maximized value of the likelihood function for the estimated model. The AIC is not a test on the model in the sense of hypothesis testing; rather, it is a tool for model selection. Given a data set, competing models may be ranked according to their AIC. In terms of interpretation, the model with the lowest AIC provides the best model fit (6). 
Further analyses were also conducted using BMI percentile and number of laps as continuous variables instead of the categorical approach reported above; however, as similar results were observed for these models, findings are not reported here. The data presented here are from the baseline data collection of a cluster-randomized controlled trial in which the effect of a multicomponent intervention is being tested to prevent risk factors for type 2 diabetes. Therefore, although to take account of the multiple tests performed $\alpha$ was set at 0.01 , all $P$ values reported within this article represent exploratory findings because the study was powered to test the effect of the intervention on the final primary outcomes only. All analyses were performed using SAS version 9.2 (SAS Institute, Cary, NC).

\section{RESULTS}

As shown in Table 1, the sample that was included in the analysis was $58.6 \%$ Hispanic, $20.8 \%$ black, and $20.7 \%$ white and included a spread of pubertal stages and socioeconomic groups (Table 1). A little more than half $(50.6 \%)$ of the participants were normal weight (BMI $<85$ th percentile) with $30.0 \%$ obese (BMI $\geq 95$ th percentile). One-way ANOVA tests indicated that there were gender differences for both fitness $(P<0.0001)$ and BMI categories $(P<0.0013)$, with higher levels of obesity among the males and lower levels of fitness among the girls. The distribution of fitness laps and the associated quintile thresholds are presented separately for males and females in Figure 1. There was an approximately even distribution of girls $(631,554,385,573$, and 471) and boys $(560,438,433,458$, and 452) within each quintile, with the slight variations in quintile size because of ties in the number of laps. The gender-specific fitness quintiles were as follows: level 1 = 0-11 laps for both males and females, level 2=12-16 laps for males and 12-15 laps for females, level $3=$ 17-24 laps for males and 16-18 laps for females, level $4=25-35$ laps for males and 19-26 laps for females, and level 5 = 36-89 laps for males and 27-74 laps for females.

Means and 95\% confidence intervals for each of the cardiometabolic risk factors are presented for BMI groups for both genders in Table 2. The mean values for each risk factor (except HDL which was the reverse) increased across the obesity groups $(P<0.001)$, with values highest for the obese group (BMI $\geq 95$ th percentile) and lowest for the normal weight group (BMI <85th percentile). Follow-up tests indicated that waist circumference, HDLcholesterol, triglycerides, diastolic blood pressure, and insulin were different among each of the three BMI groups for both genders.

Means and 95\% confidence intervals for each of the cardiometabolic risk factors are presented stratified by gender-specific fitness quintiles for both genders in Table 3. Waist circumference, LDL-cholesterol, triglycerides, diastolic blood pressure, and insulin were all inversely associated with fitness quintiles, with higher levels of fitness associated with lower risk factor means in both boys and girls. HDL-cholesterol levels were positively associated with fitness quintiles in both boys and girls, with higher levels of HDL associated with higher quintiles of fitness. Fitness quintiles were not associated with girls' total cholesterol or the systolic blood pressure and with glucose levels of either gender. Follow-up tests indicated that, with the exception of male waist circumference, there were no significant differences between the cardiometabolic risk factor levels of all quintiles. For example, for girls' LDL-cholesterol, there was only a significant difference between the lowest and top two highest quintiles of fitness. Moreover, only waist circumference and insulin were significantly different between the first and second quintiles of fitness. Similarly, very few of the comparisons between adjacent fitness quintiles (e.g., 2 vs 3 ) were significant for most of the cardiometabolic risk factors. Collectively, these findings demonstrate a pattern whereby, although cardio-metabolic risk factors differed by quintiles of fitness, differences tended to be statistically significant only when nonadjacent groups with greater differences in fitness levels were compared. 
Table 4 presents a comparison of models when BMI group, fitness group, or both BMI group and fitness group were included in models to predict cardiometabolic risk factors. When both BMI group and fitness were included in the model together, BMI group was a significant predictor of all cardiometabolic risk factors except girls' total cholesterol. In the same models, fitness was associated only with waist circumference (both genders), LDLcholesterol (males), systolic blood pressure (males), and insulin (both genders). Models that included BMI alone or both fitness and BMI had lower AIC values than models that just included fitness. In four models, AIC did not improve with the addition of fitness to models that included BMI; these models were HDL in males, triglycerides in males, triglycerides in females, and glucose in females. Because lower AIC values indicate improved model fit, the analyses demonstrate that, in all instances, including both BMI group and fitness quintiles in the analysis improved the prediction of cardiometabolic risk factors.

\section{DISCUSSION}

The data presented in this article revealed that both fitness and fatness were associated with cardiometabolic risk factors among a large, ethnically diverse sample of US sixth-grade students. Post hoc tests indicated with a few exceptions that there were significant differences in cardiometabolic risk factor levels between each of the three BMI groups, but fitness-based differences tended to be significant only when non-adjacent fitness groups were compared. It is also important to highlight that several associations between fitness and cardiometabolic risk factors were attenuated after adjustment for fatness, indicating that fatness is likely to be the more dominant predictor of cardiometabolic risk factors at this age.

These findings are consistent with several previous childhood studies $(10,11)$ including a recent article that reported that fatness was a stronger predictor of cardiometabolic risk factors than fitness among a cohort white and African American youth from North Carolina (27). The data presented here extend those findings by demonstrating in a unique, ethnically diverse cohort of adolescents recruited from across the United States that fatness is a stronger predictor of cardiometabolic risk factors than fitness among youth. The data also show that although cardiometabolic risk levels increased with each BMI group, the fitness differences tended to only be evident when nonsequential fitness quintiles were compared. Similar findings have also been observed in data from the Aerobics Center Longitudinal Study, where the differences in metabolic risk score were accounted for by BMI levels but not by fitness in males aged 8-18 yr (12). Collectively, these findings indicate that, whereas preventing weight gain and improving fitness are both likely to be helpful in maintaining lower levels of cardiometabolic risk factors among youth, greater emphasis on obesity prevention is warranted.

Comparison of the data presented in this study and published adult studies suggests that the importance of fitness changes with age and the development of lifestyle diseases such as type 2 diabetes. The Look Ahead study of overweight or obese adults with type 2 diabetes reported that, among females, fitness was a significant predictor of triglycerides but BMI was not and that only fitness predicted the Framingham risk factor scores for both genders (41). Comparison of the studies might suggests that, among a disease-free sample of young adolescents, fatness is more closely associated with cardiometabolic risk factors than aerobic fitness, but associations may change with the development of obesity and type 2 diabetes mellitus. Fat cell size increases during obesity and is likely to have a direct effect on pathophysiology and, therefore, also risk factor profile. Because fitness is heavily influenced by genetic factors (3), it may take a while for the well-established associations between adult fitness and cardiometabolic risk factors and mortality risk to develop, only becoming evident in adulthood (22,32). It is, however, important to acknowledge that, in the present study, only $0.2 \%$ males and $2.5 \%$ females would be categorized as above average fitness using the 
FITNESSGRAM cutoff criteria (40). Therefore, to assess associations with a range of fitness levels, we created gender-specific fitness quintiles instead of using the dichotomous FITNESSGRAM criteria in our analysis. This approach limits our ability to draw conclusions about "fit" versus "unfit" adolescents. In addition, the low levels of fitness and the lack of highly fit adolescents could have attenuated the relationship between fitness and the cardiometabolic risk factors.

Consistent with previous studies, the girls in this study had lower levels of fitness than boys $(19,34)$. Therefore, although the associations between fitness and cardiometabolic risk reported here suggest a need to develop interventions aimed at maintaining adolescent fitness, there is a particular need to develop strategies to increase girls' fitness. This is particularly important because low levels of fitness track into adulthood (35), thus increasing the importance that children, especially girls, develop habits to promote and maintain fitness that will benefit them later in life.

\section{Strengths and limitations}

This study has extended the current literature base by providing new information about the associations among fitness, obesity, and cardiometabolic risk factors among a large, ethnically diverse sample of sixth-grade youth. This study does, however, have some limitations that need to be recognized. Although the 20-MST has been strongly associated $(r$ $=0.69-0.96)(2,26,36)$ with aerobic fitness in laboratory tests, the 20-MST does not directly measure aerobic fitness. Validity of estimated aerobic fitness is largely influenced by levels of maximal exertion or motivation of participants. Therefore, 20-MST only provides an estimate of fitness. The study is also limited by the single age group, which precludes an assessment of age or maturation-related differences in associations. It is also important to recognize that although a validated scale was used, pubertal stage was self-reported by the participants and could therefore be prone to a degree of measurement error. Finally, because the study is cross-sectional, it is not possible to identify directions of associations or causation between variables of interest.

In conclusion, the data presented in this article have shown that, among a large, ethnically diverse cohort of US youth, both fatness and fitness are associated with cardiometabolic risk factors. Associations were, however, stronger for fatness than fitness, and several fitness associations were attenuated after controlling for fatness. Results, therefore, suggest that although maintaining high levels of fitness and preventing obesity may positively affect cardiometabolic risk factors, the greater benefit may be obtained from obesity prevention, and there is a need to redouble efforts in this area.

\section{Acknowledgments}

This work was completed with funding from National Institute of Diabetes and Digestive and Kidney Diseases/ National Institutes of Health grant numbers U01-DK61230, U01-DK61249, U01-DK61231, and U01-DK61223 to the STOPP-T2D collaborative group, with additional support from the American Diabetes Association.

\section{REFERENCES}

1. American Medical Association. Expert Committee Recommendations on the Assessment, Prevention, and Treatment of Child and Adolescent Overweight and Obesity [Internet]. American Medical Association; Washington (DC): [2007 Nov 2]. Available from: http://www.ama-assn.org/ama1/pub/upload/mm/433/ped_obesity_recs.pdf

2. Boreham CA, Paliczka VJ, Nichols AK. A comparison of the PWC170 and 20-MST tests of aerobic fitness in adolescent schoolchildren. J Sports Med Phys Fitness 1990;30(1):19-23. [PubMed: 2366530] 
3. Bouchard C, Dionne FT, Simoneau J-A, Boulay MR. Genetics of aerobic and anaerobic performance. Exerc Sport Sci Rev 1992;20:27-58. [PubMed: 1623888]

4. Brage S, Wedderkopp N, Ekelund U, et al. Features of the metabolic syndrome are associated with objectively measured physical activity and fitness in Danish children: the European Youth Heart Study. Diabetes Care 2004;27(9):2141-8. [PubMed: 15333475]

5. Brunzell JD, Davidson M, Furberg CD, et al. Lipoprotein management in patients with cardiometabolic risk: consensus conference report from the American Diabetes Association and the American College of Cardiology Foundation. J Am Coll Cardiol 2008;51(15):1512-24. [PubMed: 18402913]

6. Burnham KP, Anderson DR. Multi-model inference: understanding AIC and BIC in model selection. Sociol Methods Res 2004;33:261-304.

7. Buse J, Hirst K. The HEALTHY Study: introduction. Int J Obes (Lond) 2009;33(4 suppl):S1-2. [PubMed: 19623183]

8. Centers for Disease Control National Center for Health Statistics [Internet]. CDC Growth Charts for the United States. Centers for Disease Control; Atlanta (GA): 2000 [2009 Jun 1]. Available from: http://www.cdc.gov/growthcharts

9. Centers for Disease Control National Center for Health Statistics [Internet]. A SAS Program for the CDC Growth Charts. Centers for Disease Control; Atlanta (GA): [2009 Jun 1]. Available from: http://www.cdc.gov/nccdphp/dnpa/growthcharts/sas.htm

10. Cook S, Auinger P, Li C, Ford ES. Metabolic syndrome rates in United States adolescents, from the National Health and Nutrition Examination Survey, 1999-2002. J Pediatr. 2008 152(2):16570.

11. Eisenmann JC. Aerobic fitness, fatness and the metabolic syndrome in children and adolescents. Acta Paediatr 2007;96(12):1723-9. [PubMed: 17971189]

12. Eisenmann JC, Welk GJ, Wickel EE, Blair SN. Combined influence of cardiorespiratory fitness and body mass index on cardiovascular disease risk factors among 8-18 year old youth: the Aerobics Center Longitudinal Study. Int J Pediatr Obes 2007;2(2):66-72. [PubMed: 17763013]

13. Ekelund U, Anderssen SA, Froberg K, Sardinha LB, Andersen LB, Brage S. Independent associations of physical activity and cardiorespiratory fitness with metabolic risk factors in children: the European youth heart study. Diabetologia 2007;50(9):1832-40. [PubMed: 17641870]

14. Fridewald WT, Levy RI, Fredrickson DS. Estimation of the concentration of low-density lipoprotein cholesterol in plasma, without use of the preparative ultracentrifuge. Clin Chem 1972;18:499-502. [PubMed: 4337382]

15. Froberg K, Andersen LB. Mini review: physical activity and fitness and its relations to cardiovascular disease risk factors in children. Int J Obes (Lond) 2005;29(2 suppl):S34-9. [PubMed: 16385750]

16. Gutin B, Yin Z, Humphries MC, Barbeau P. Relations of moderate and vigorous physical activity to fitness and fatness in adolescents. Am J Clin Nutr 2005;81(4):746-50. [PubMed: 15817847]

17. Hainline, AJ.; Karon, J.; Kippel, K. Manual of Laboratory Operations: Lipid Research Clinics Program, Lipid and Lipoprotein Analysis. 2nd ed.. US Department of Health \& Human Services; Washington (DC): 1983. p. 75-628.

18. Hirst K, Baranowski T, DeBar L, et al. HEALTHY Study rationale, design and methods: moderating risk of type 2 diabetes in multi-ethnic middle school students. Int J Obes (Lond) 2009;33(4 suppl):S4-20. [PubMed: 19623188]

19. Hussey J, Bell C, Bennett K, O'Dwyer J, Gormley J. Relationship between the intensity of physical activity, inactivity, cardiorespiratory fitness and body composition in 7-10-year-old Dublin children. Br J Sports Med 2007;41(5):311-6. [PubMed: 17395610]

20. Jago R, Harrell JS, McMurray RG, Edelstein S, El Ghormli L, Bassin S. Prevalence of abnormal lipid and blood pressure values among an ethnically diverse population of eighth-grade adolescents and screening implications. Pediatrics 2006;117(6):2065-73. [PubMed: 16740849]

21. Lee CD, Blair SN. Cardiorespiratory fitness and stroke mortality in men. Med Sci Sports Exerc 2002;34(4):592-5. [PubMed: 11932565]

22. Lee CD, Blair SN, Jackson AS. Cardiorespiratory fitness, body composition, and all-cause and cardiovascular disease mortality in men. Am J Clin Nutr 1999;69:373-80. [PubMed: 10075319] 
23. Lee DC, Sui X, Church TS, Lee IM, Blair SN. Associations of cardiorespiratory fitness and obesity with risks of impaired fasting glucose and type 2 diabetes in men. Diabetes Care 2009;32(2):25762. [PubMed: 18984778]

24. Leger LA, Lambert J. A maximal multistage $20-\mathrm{m}$ shuttle run test to predict $\mathrm{VO}_{2 \max }$. Eur J Appl Physiol Occup Physiol 1982;49(1):1-12. [PubMed: 7201922]

25. Leger LA, Mercier D, Gadoury C, Lambert J. The multistage 20 metre shuttle run test for aerobic fitness. J Sports Sci 1988;6(2):93-101. [PubMed: 3184250]

26. Liu NY, Plowman SA, Looney MA. The reliability and validity of the 20-meter shuttle test in American students 12 to 15 years old. Res Q Exerc Sport 1992;63(4):360-5. [PubMed: 1439160]

27. Ondrak KS, McMurray RG, Bangdiwala SI, Harrell JS. Influence of aerobic power and percent body fat on cardiovascular disease risk in youth. J Adolesc Health 2007;41(2):146-52. [PubMed: 17659218]

28. Pate RR, Wang CY, Dowda M, Farrell SW, O'Neill JR. Cardiorespiratory fitness levels among US youth 12 to 19 years of age: findings from the 1999-2002 National Health and Nutrition Examination Survey. Arch Pediatr Adolesc Med 2006;160(10):1005-12. [PubMed: 17018458]

29. Petersen AC, Crockett L, Richards M, Boxer A. A self-report measure of pubertal status: reliability, validity, and initial norms. J Youth Adolesc 1988;17:117-33.

30. Ruiz JR, Ortega FB, Rizzo NS, et al. High cardiovascular fitness is associated with low metabolic risk score in children: the European Youth Heart Study. Pediatr Res 2007;61(3):350-5. [PubMed: 17314696]

31. STOPP-T2D Prevention Study Group. Presence of diabetes risk factors in a large U.S. eighth-grade cohort. Diabetes Care 2006;29(2):212-7. [PubMed: 16443862]

32. Sui X, Hooker SP, Lee IM, et al. A prospective study of cardiorespiratory fitness and risk of type 2 diabetes in women. Diabetes Care 2007;31(3):550-5. [PubMed: 18070999]

33. Tanner, JM. Growth at Adolescence. Blackwell; Oxford (UK): 1962. p. 340

34. Treuth MS, Hou N, Young DR, Maynard LM. Accelerometry-measured activity or sedentary time and overweight in rural boys and girls. Obes Res 2005;13(9):1606-14. [PubMed: 16222064]

35. Twisk JWR, Kemper HCG, Van Mechelen W. The relationship between physical fitness and physical activity during adolescence and cardiovascular disease risk factors at adult age. The Amsterdam Growth and Health Longitudinal Study. Int J Sports Med 2002;23(suppl):S8-14. [PubMed: 12012256]

36. van Mechelen W, Hlobil H, Kemper HCG. Validation of two running tests as estimates of maximal aerobic power in children. Eur J Appl Physiol Occup Physiol 1986;55(5):503-6. [PubMed: 3769907]

37. Warnick GR. Enzymatic methods for quantification of lipoprotein lipids. Methods Enzymol 1986;129:101-23. [PubMed: 3724535]

38. Warnick GR, Knopp RH, Fitzpatrick V, Branson L. Estimating low-density lipoprotein cholesterol by the Fridewald equation is adequate for classifying patients on the basis of nationally recommended cutpoints. Clin Chem 1990;36:15-9. [PubMed: 2297909]

39. Weir MR, Stafford EM, Gregory G, Lawson MA, Pearl W. The relationship between sexual maturity rating, age and increased blood pressure in adolescents. J Adolesc Health Care 1988;9:465-9. [PubMed: 3182359]

40. Welk, GJ.; Meredith, MD. Fitnessgram/Activitygram Reference Guide. The Cooper Institute; Dallas (TX): 2008. p. 96-121.

41. Wing RR, Jakicic J, Neiberg R, et al. Fitness, fatness, and cardiovascular risk factors in type 2 diabetes: look ahead study. Med Sci Sports Exerc 2007;39(12):2107-16. [PubMed: 18046181] 


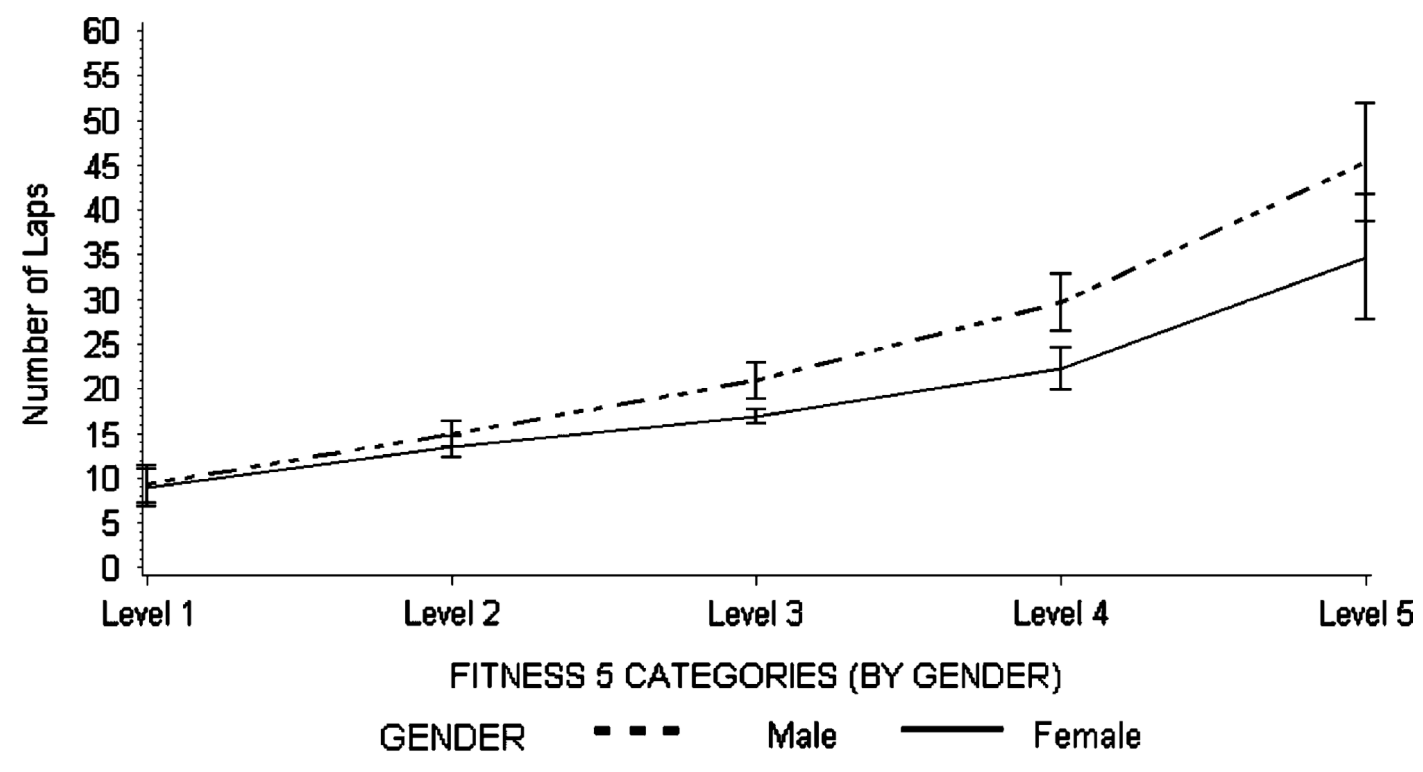

Level 1: Males $N=560$, Females $N=631$; Level 2: Males $N=438$, Females $N=554$; Level 3: Males $N=433$, Females $N=385$; Level 4: Males $N=458$, Females $N=573$; Level 5: Males $N=452$, Females $N=471$

FIGURE 1.

Fitness and quintile break points for males and female adolescents in the HEALTHY Study. 


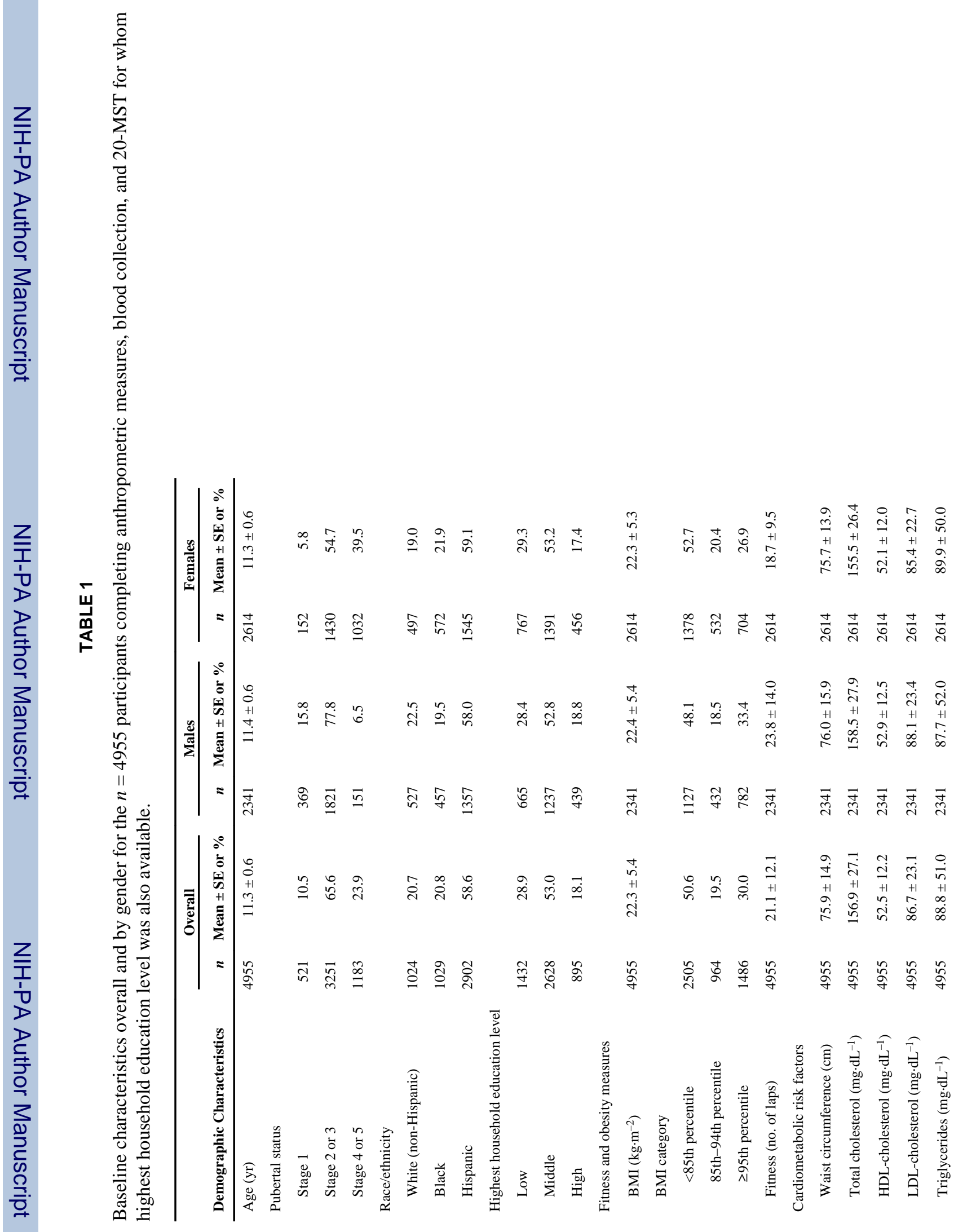




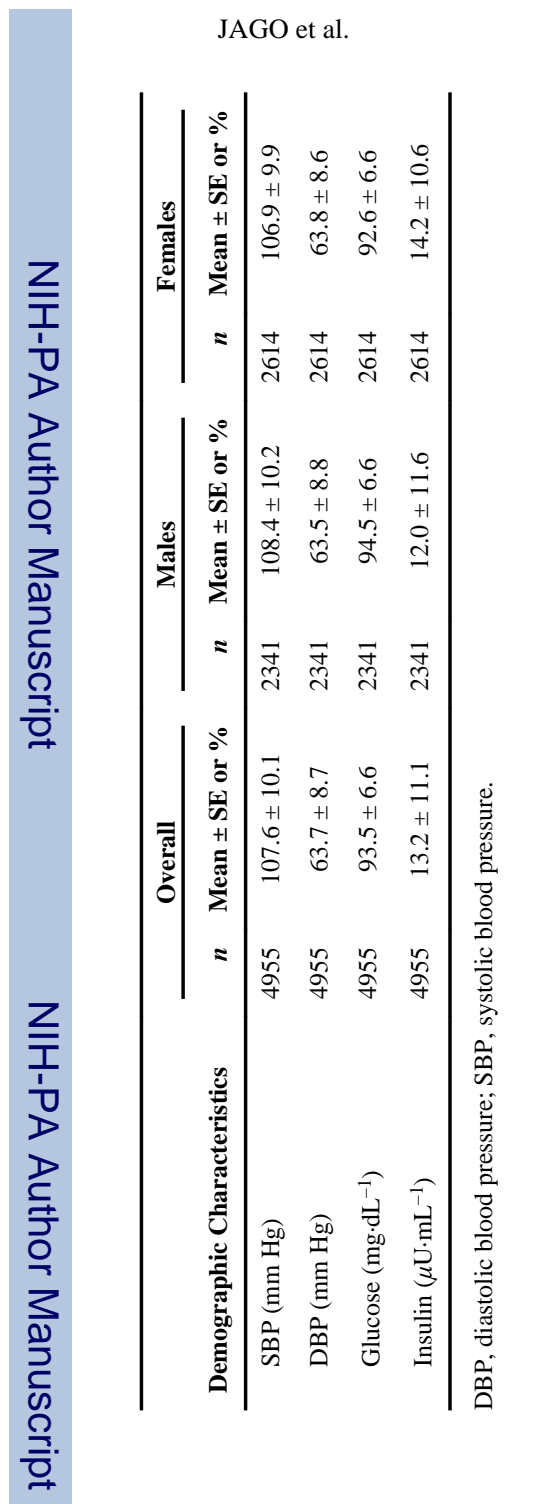

Page 13 


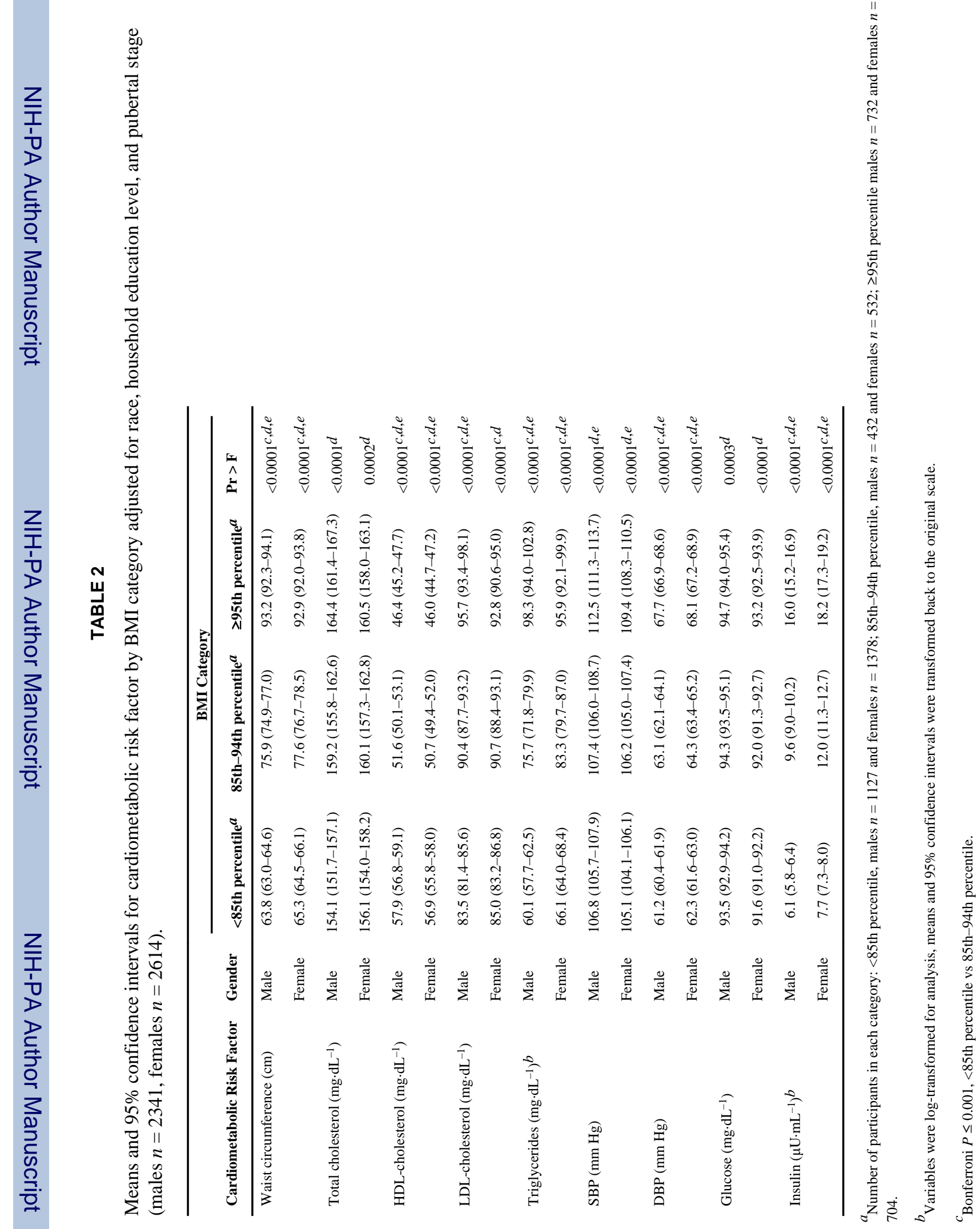




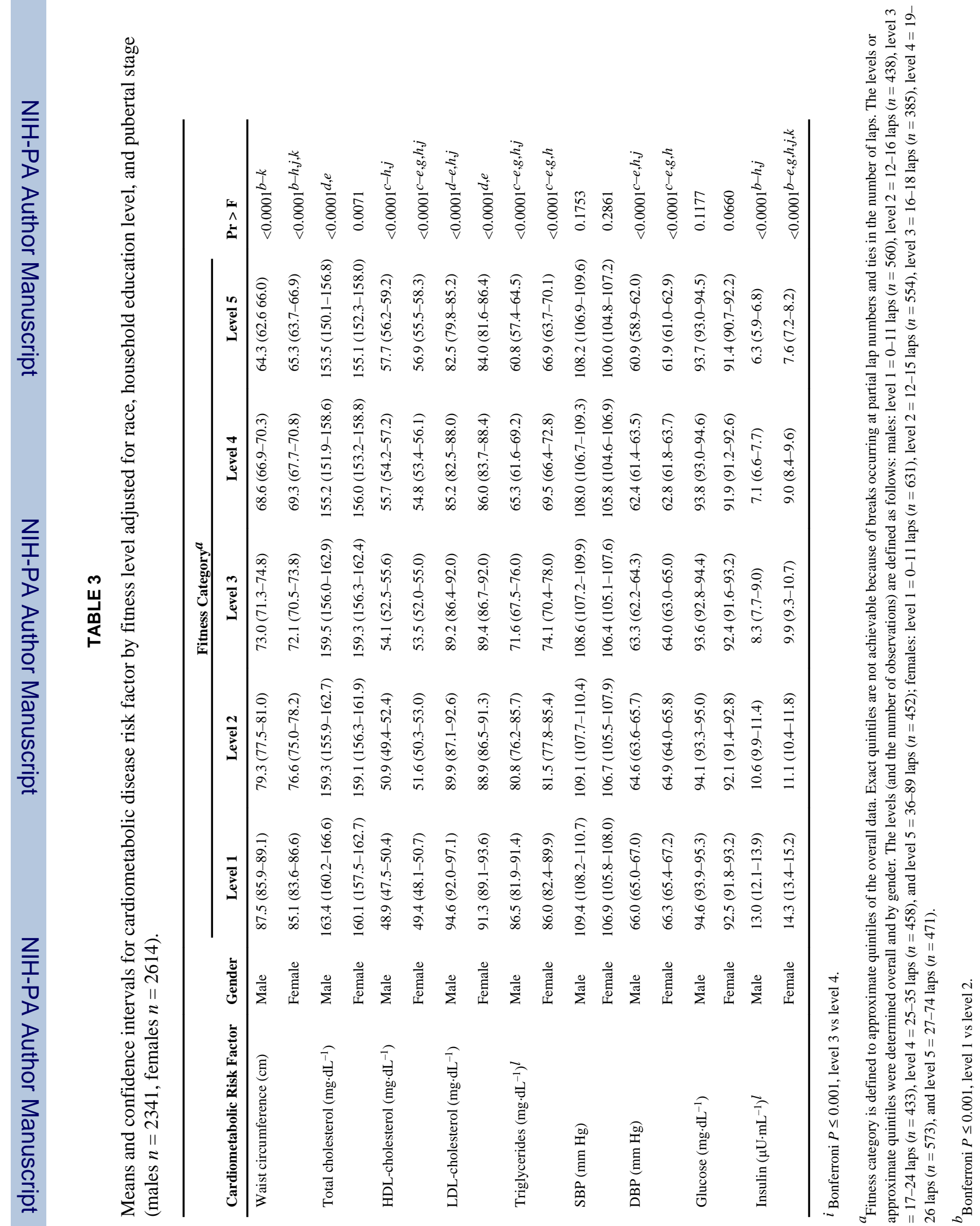




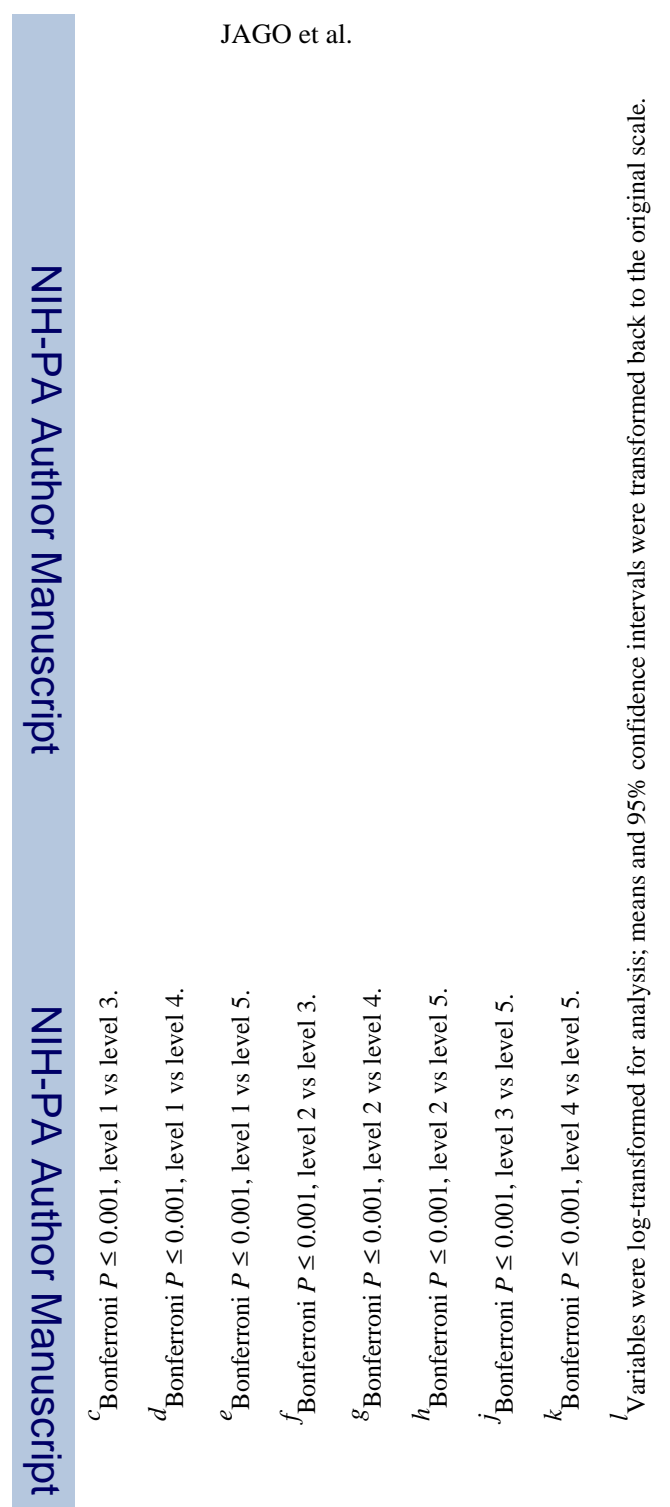

Med Sci Sports Exerc. Author manuscript; available in PMC 2010 August 13. 


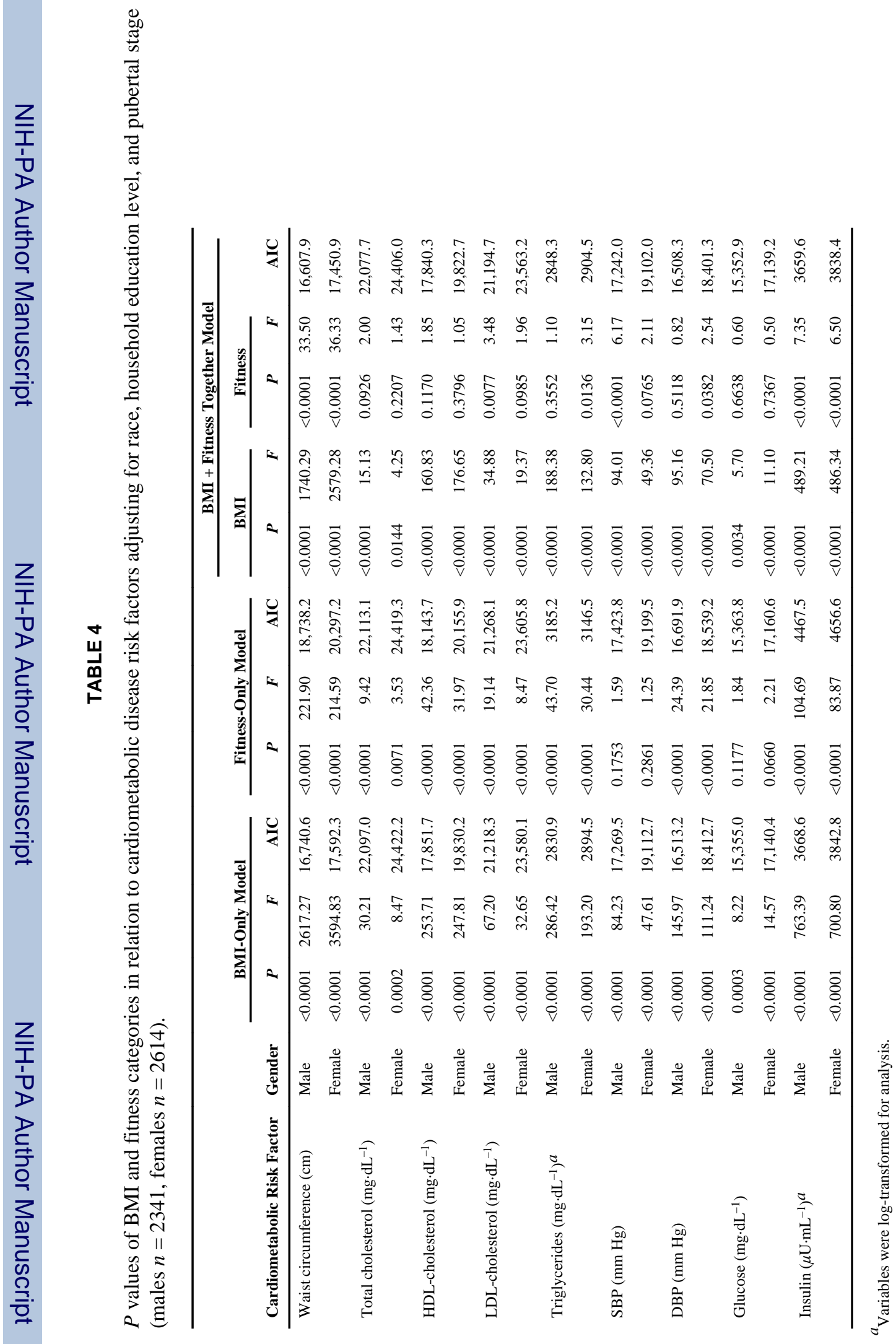

